\title{
Influence of variable viscosity and wall properties on the peristalsis of Jeffrey fluid in a curved channel with radial magnetic field
}

\author{
Manjunatha Gudekote ${ }^{1}$, Divya Baliga $\mathbf{B}^{1}$, Rajashekhar Choudhari ${ }^{1,2, *}$, \\ Hanumesh Vaidya ${ }^{3}$, K.V. Prasad ${ }^{3}$, O.D. Makinde ${ }^{4}$ \\ ${ }^{1}$ Department of Mathematics, Manipal Institute of Technology, Manipal Academy of Higher \\ Education, Manipal, Karnataka, India-576104 \\ ${ }^{2}$ Bhaskaracharya Study Chair, Karnataka State Akkamahadevi Women's University, Vijayapura, \\ Karnataka, India-586108 \\ ${ }^{3}$ Department of Mathematics, Vijayanagara Srikrishnadevaraya University, Ballari, Karnataka, \\ India-583105 \\ ${ }^{4}$ Faculty of Military Science, Stellenbosch University, Private Bag X2, Saldanha 7395, South Africa
}

Received: 25 March 2020; Received in revised form: 20 May 2020; Accepted: 23 May 2020;

Published online 2 June 2020

(C) Published at www.ijtf.org

\begin{abstract}
The current investigation attempts to address the peristalsis exhibited by a Jeffrey fluid through channels with curvature and compliant walls. The flow of fluid is exposed to an external magnetic field. Moreover, variation of the viscosity of the fluid with the spatial coordinate is considered. Long wavelength and small values of Reynolds number are considered for the mathematical modeling of the problem under scope. The system of differential equations thus obtained is non-linear, the solution for which is obtained by the method of perturbation for small values of variable viscosity. The authors have provided special emphasis on the influence of pertinent parameters on velocity and trapping phenomenon. The results obtained suggest that as the channel changes from straight to curved, the velocity profile bends away from the center of the channel. Further, the trapped bolus volume is seen to be reducing with decrease in the Hartmann number.
\end{abstract}

Keywords: Curved channel; Hartmann number; Jeffrey parameter; peristalsis; variable viscosity.

\section{Introduction}

Peristaltic mechanism is the mode of transport of various materials by the continuous contracting and relaxing waves moving along the walls of a distensible tube. This mechanism facilitates the transportation of various biofluids, for example, the motion of chyme, transport of the food bolus through the esophagus, movement of spermatozoa and circulation of blood 
Manjunatha et al.

International Journal of Thermofluid Science and Technology (2020), Volume 7, Issue 2, Paper No. 070203

through the arteries. Owing to its applications in industrial and medical fields, peristalsis has gained significant importance among researchers in the past few years. Among the various fluid models available, the usage of non-Newtonian models is more advantageous in physiology and industries. Inspired by this, the first investigation on peristaltic mechanism for a non-Newtonian fluid was carried out by Raju and Devanathan using the power law model [1]. Following this, substantial research has been conducted for various models representing the non-Newtonian fluids. One such model, the Herschel-Bulkley model, was used by Rajashekhar et al. [2]. They observed that for smaller values of yield stress, the power law and Herschel-Bulkley models show similar behavior. Effects of heat transfer on an inclined tube for a Bingham fluid with different peristaltic wave forms were studied by Vaidya et al. [3,4]. For non-Newtonian fluids, heat transfer properties have been investigated with the considerations of slip conditions and wall properties [5-7].

\begin{tabular}{|llll|}
\hline \multicolumn{2}{l}{ Nomenclature } & \multicolumn{2}{l|}{} \\
$a^{\prime}$ & wave amplitude & $t$ & time \\
$B_{0}$ & applied magnetic field & $u$ & dimensionless radial velocity \\
$c$ & wave speed & $w$ & dimensionless axial velocity \\
$C_{f}$ & skin-friction coefficient & $x$ & dimensionless axial distance \\
$d^{\prime}$ & half-width of the curved channel & Greek symbols \\
$E_{1}$ & elastic tension in the walls & $\delta$ & wave number \\
$E_{2}$ & mass per unit area & $\varepsilon$ & amplitude ratio \\
$E_{3}$ & coefficient of viscous damping & $\lambda$ & wavelength \\
$H$ & Hartmann number & $\lambda$ & Jeffrey parameter \\
$k$ & curvature parameter & $\mu(r)$ & viscosity varying with channel width \\
$p$ & dimensionless pressure & $\phi$ & coefficient of variable viscosity \\
$r$ & dimensionless radial distance & $\psi$ & dimensionless streamline function \\
$r^{*}$ & radius of the curved channel & $\rho$ & fluid density \\
$\operatorname{Re}$ & Reynolds number & $\sigma$ & electrical conductivity of the fluid \\
\hline
\end{tabular}

In the aforementioned studies, the mechanics of peristalsis have been studied for fluids flowing through different two-dimensional, symmetric and axisymmetric planar and straight channels. However, this turns out to be inadequate for physiological conduits and glandular ducts where the geometries are curved, and curvatures have considerable impact on the fluid flow. In studies on industrial and physical processes, the curvilinear coordinates of the curved channel lead to complex mathematical computations. This has motivated many studies on peristalsis through a curved channel. The peristaltic mechanism of a viscous incompressible fluid was investigated by Ali et al. [8] where the fluid flow was considered through a rectangular curved channel. Extending upon their initial investigations, they also considered peristalsis for a third-grade non-Newtonian fluid in a curved channel, where it was found that the trapped bolus lost its symmetry in a curved channel and divides itself into two asymmetrical parts [9]. Through studies conducted on Newtonian fluids with focus on heat transfer through a curved channel, it was found that the heat transfer occurred at a slower rate than in a straight channel [10]. Investigations conducted on the transfer of heat 
Manjunatha et al.

International Journal of Thermofluid Science and Technology (2020), Volume 7, Issue 2, Paper No. 070203

and mass during the peristaltic mechanism of various non-Newtonian fluids within curved channels with compliant walls and suitable slip and no-slip conditions are found in the literature [11-14].

The consideration of magnetic field in industrial applications as well as biomedical engineering has garnered the interest of many researchers in the magnetohydrodynamic flows. In addition, magnetohydrodynamics has proved to be extremely useful during surgeries in reducing the bleeding, treatment of cancers, removal of the arterial blockages, and extraction of molten metals from non-metallic substrate among other applications. Recent developments in the field of medicines have made it possible for the magnetic flux to increase the effectiveness and precision in the drug delivery systems. Influenced by the widespread applications of magnetic field applied externally and the realistic usage of curved channels, studies have been conducted on the magnetohydrodynamics of peristaltic mechanism through curved geometries. Hayat et al. [15] considered a third-grade fluid in a compliant walled curved channel to examine the Dufour and Soret effects. In their studies, they observed that the Dufour and Soret numbers have an increasing effect on concentration and temperature profiles respectively. The combined effect of slip conditions and radial magnetic field was explored by Shehzad et al. [16]. Their results indicated that in curved conduits, the velocity profiles along the axis were asymmetric about its central line. Further, effects of magnetic fields on the peristaltic flow of a third-grade fluid through a curved channel were studied by Hayat et al. [17]. The MHD peristaltic transport of various Newtonian and non-Newtonian fluids through curved channel have been studied with varying boundary conditions [18-24].

In the studies mentioned above, the physical fluid properties, especially viscosity, are considered constant and independent of the spatial variables. This assumption fails in giving better insight to the peristaltic mechanism in the digestive tract, bloodstream in vessels of small radius, just to mention a few, where the viscosity is found to be varying across the thickness of the channel [25-30]. Owing to this, Vaidya et al. [31] examined the impact of variable liquid properties on peristalsis observed for a Rabinowitsch fluid within a uniform compliant-walled channel. Their results indicate that variable viscosity enhances the fluid velocity. It was also found that an increased variable viscosity led to an increase in the coefficient of skin friction for dilatant fluids. However, pseudoplastic and Newtonian fluids displayed decreased coefficient of skin friction with increased variable viscosity. Manjunatha et al. [32,33] have investigated the influence of variable liquid properties of a Jeffrey fluid through a uniform channel as well as elastic tube subject to porous boundary conditions. The concept of variable viscosity has also been extended for peristaltic mechanisms in the curved channel by Hayat et al. [34] in their magnetohydrodynamic studies of Bingham plastic fluid.

The above studies indicate that an investigation on the peristalsis of a non-Newtonian fluid which exhibits variable viscosity and is flowing through a curved channel is very essential to completely understand the behavior of many biological and physiological fluids. As per the author's knowledge, very little studies have been conducted in this direction. Thus, the current work intends to showcase the impact of varying viscosity of Jeffrey fluid flowing through channel with significant curvature, which is exposed to a radial magnetic field. The governing differential equations, after using small Reynolds number and long wavelength approximations, are solved through perturbation method for small values of variable viscosity. Further, axial velocity profiles, pressure rise versus flow rate, skin friction coefficient and trapping phenomenon are plotted and analyzed for different values of the pertinent parameters. 


\section{Mathematical Model of the Problem}

The half width of the curved channel is assumed to be $d^{\prime}$. The channel is converted into a circle with center $O$ denoted by and radius denoted by $r^{*}$. The behavior of the fluid is defined by the Jeffrey fluid model and the flow is generated by the movement of sinusoidal waves of velocity $c$ along the flexible walls of the curved channel. The radial direction of fluid flow is $r$, while the axial direction is denoted by $x$. The velocity components in the radial and axial directions are $u(r, x, t)$ and $v(r, x, t)$ respectively. The fluid flow is subjected to an external radial magnetic field (see Figure 1).

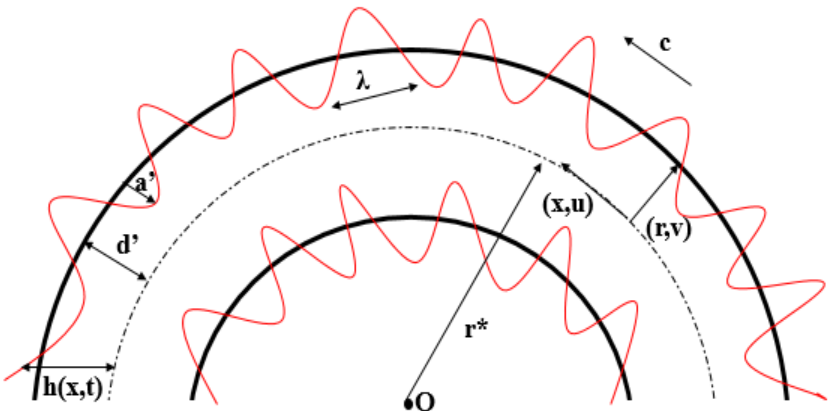

Fig. 1. Geometry of the problem.

The geometry of the peristaltic wave is given by:

$$
r= \pm h(x, t)= \pm\left[d^{\prime}+a^{\prime} \sin \left(\frac{2 \pi}{\lambda}(x-c t)\right)\right],
$$

where $\pm h$ indicates the displacements of the upper and lower walls respectively.

The fluid becomes electrically conducting when it is subjected to a radial magnetic field $\mathbf{B}$, given by

$$
\mathbf{B}=\left(\frac{B_{0}}{r+r^{*}}, 0,0\right) .
$$

From Ohm's law, we have

$$
\mathbf{J} \times \mathbf{B}=\left(0, \frac{-\sigma B_{0}{ }^{2} u}{\left(r+r^{*}\right)^{2}}, 0\right),
$$

where $\mathbf{J}$ is the current density.

For the problem under consideration, the equations of conservation of momentum and mass are given below

$$
\frac{\partial v}{\partial r}+\frac{r^{*}}{r+r^{*}} \frac{\partial u}{\partial x}+\frac{v}{r+r^{*}}=0
$$




$$
\begin{aligned}
\rho\left[\frac{\partial v}{\partial t}+v \frac{\partial v}{\partial r}+\frac{r^{*} u}{r^{*}+r} \frac{\partial v}{\partial x}-\frac{u^{2}}{r^{*}+r}\right]=-\frac{\partial p}{\partial r}-\frac{S_{x x}}{r^{*}+r}+\frac{1}{r^{*}+r} \frac{\partial}{\partial r}\left[\left(r^{*}+r\right) S_{r r}\right] \\
+\frac{r^{*}}{r^{*}+r} \frac{\partial S_{x r}}{\partial x}, \\
\rho\left[\frac{\partial u}{\partial t}+v \frac{\partial u}{\partial r}+\frac{r^{*} u}{r^{*}+r} \frac{\partial u}{\partial x}+\frac{u v}{r^{*}+r}\right]=-\frac{r^{*}}{r^{*}+r} \frac{\partial p}{\partial x}+\frac{1}{\left(r^{*}+r\right)^{2}} \frac{\partial}{\partial r}\left[\left(r^{*}+r\right)^{2} S_{r x}\right] \\
+\frac{r^{*}}{r^{*}+r} \frac{\partial S_{x x}}{\partial x}-\frac{\sigma B_{0}{ }^{2} u}{\left(r^{*}+r\right)^{2}} .
\end{aligned}
$$

where $\tau^{*}, m^{*}$ and $C^{*}$ are the elastic parameters of the wall (elastic tension in the walls, mass per unit area and the coefficient of viscous damping respectively).

The components of the extra stress tensor $\mathbf{S}$ for a Jeffrey fluid are given

by

$$
S_{x r}=\frac{\mu(r)}{1+\lambda_{1}}\left[1+\frac{\delta \lambda_{2} c}{d^{\prime}}\left(\frac{-\partial \psi}{\partial r} \frac{\partial}{\partial x}-\frac{\partial \psi}{\partial x} \frac{\partial}{\partial r}\right)\right]\left(-\frac{\partial^{2} \psi}{\partial r^{2}}-\delta^{2} \frac{\partial^{2} \psi}{\partial x^{2}}\right),
$$

$$
\begin{aligned}
& S_{x x}=\frac{-2 \delta \mu(r)}{1+\lambda_{1}}\left[1+\frac{\delta \lambda_{2} c}{d^{\prime}}\left(\frac{-\partial \psi}{\partial r} \frac{\partial}{\partial x}-\frac{\partial \psi}{\partial x} \frac{\partial}{\partial r}\right)\right] \frac{\partial^{2} \psi}{\partial x \partial r} \\
& S_{r r}=\frac{2 \delta \mu(r)}{1+\lambda_{1}}\left[1+\frac{\delta \lambda_{2} c}{d^{\prime}}\left(-\frac{\partial \psi}{\partial r} \frac{\partial}{\partial x}-\frac{\partial \psi}{\partial x} \frac{\partial}{\partial r}\right)\right] \frac{\partial^{2} \psi}{\partial x \partial r}
\end{aligned}
$$

where $\delta=\frac{d^{\prime}}{\lambda}$ is the wavenumber and $\lambda_{1}$ and $\lambda_{2}$ are the Jeffrey parameters.

The boundary conditions are as described below:

$$
\begin{aligned}
& u=0, \text { at } r= \pm h \\
& \frac{\partial p}{\partial x}=\frac{\partial}{\partial x}\left[-\tau^{*} \frac{\partial^{2}}{\partial x^{2}}+m^{*} \frac{\partial^{2}}{\partial t^{2}}+C^{*} \frac{\partial}{\partial t}\right]
\end{aligned}
$$

The above dimensional parameters are rendered non-dimensional by the following:

$$
\begin{aligned}
& \psi^{*}=\frac{\psi}{c d^{\prime}}, x^{*}=\frac{x}{\lambda}, r^{*}=\frac{r}{d^{\prime}}, t^{*}=\frac{c t}{\lambda}, u=\frac{u^{*}}{c}, v=\frac{v^{*}}{c}, h^{*}=\frac{h}{d^{\prime}}, k=\frac{r^{*}}{d^{\prime}}, p^{*}=\frac{d^{\prime 2} p}{\mu(r) c \lambda}, \\
& \varepsilon=\frac{a^{\prime}}{d^{\prime}}, S_{i j}{ }^{*}=\frac{d^{\prime} S_{i j}}{c \mu(r)}, \operatorname{Re}=\frac{\rho c d^{\prime}}{\mu(r)}, H^{2}=\frac{\sigma B_{0}{ }^{2}}{\mu(r)}, E_{1}=\frac{-\tau^{*} d^{\prime 3}}{\lambda^{3} \rho c \mu(r)}, E_{2}=\frac{m^{*} c d^{\prime 3}}{\lambda^{2} \rho \mu(r)}, \\
& E_{3}=\frac{C^{*} d^{\prime 3}}{\lambda^{2} \rho \mu(r)},
\end{aligned}
$$


where the meaning of the non-dimensional parameters are mentioned in the nomenclature section.

We introduce the stream function $\psi(x, r, t)$ as

$$
u=-\frac{\partial \psi}{\partial r}, v=\frac{r^{*}}{r^{*}+r} \frac{\partial \psi}{\partial x} \text {. }
$$

On using the above dimensionless parameters and the assumptions of long wavelength and low Reynolds number, we obtain (on dropping the asterisk for simplicity)

$$
\begin{aligned}
& \frac{\partial p}{\partial r}=0, \\
& \frac{\partial p}{\partial x}=\frac{1}{k(r+k)} \frac{\partial}{\partial r}\left[(r+k)^{2} S_{r x}\right]+H^{2} \frac{\psi_{r}}{k(r+k)}, \\
& h=1+\varepsilon \sin [2 \pi(x-t)], \\
& \psi_{r}=0 \text { at } r= \pm h, \\
& \frac{k}{r+k}\left[E_{1} \frac{\partial^{3} h}{\partial x^{3}}+E_{2} \frac{\partial^{3} h}{\partial x \partial t^{2}}+E_{3} \frac{\partial^{2} h}{\partial x \partial t}\right]=\frac{1}{(r+k)^{2}} \frac{\partial}{\partial r}\left[(r+k)^{2} S_{r x}\right]+H^{2} \frac{\psi_{r}}{k(r+k)}, \\
& \text { (18) } S_{r x}=\frac{-\mu(r)}{1+\lambda_{1}} \frac{\partial^{2} \psi}{\partial r^{2}} .
\end{aligned}
$$

From Eqs. (14) and (15), we obtain

$$
\frac{\partial}{\partial r}\left[\frac{-1}{(r+k)} \frac{\partial}{\partial r}\left(\frac{\mu(r)}{1+\lambda_{1}}(r+k)^{2} \frac{\partial^{2} \psi}{\partial r^{2}}\right)\right]+H^{2} \frac{\psi_{r}}{k(r+k)}=0 .
$$

Here, $\mu(r)$ is the variable viscosity which is expressed as

$$
\mu(r)=e^{-\phi r}=1-\phi r+O\left(\phi^{2}\right), \quad \phi<<1,
$$

where $\phi$ is the coefficient of variable viscosity.

The choice of $\mu(r)$ (variable viscosity) here is justified physiologically because normal person or animal of similar size takes $1-2 \mathrm{~L}$ of the fluid every day. Also $6-7 \mathrm{~L}$ of the fluid is received by the small intestine as secretions from salivary glands, stomach, pancreas, liver and small intestine itself. This indicates the dependence of fluid concentration upon the spatial coordinate $r$ and hence the choice of $\mu$ in the present analysis is appropriate [35].

\section{Solution to the Problem}

The non-linear system Eq. (20) consisting of the boundary conditions given by Eqs. (17) and (18) is solved for $\psi$ (stream function) by perturbation technique for small values of the variable viscosity $\phi$. The solution is found up to the first order. As $k \rightarrow \infty$, the results are in good agreement with the studies available in the literature for a planar channel.

The perturbed series solution for $\psi$ is considered as 


$$
\psi=\psi_{0}+\phi \psi_{1}+O\left(\phi^{2}\right)
$$

On substituting the above in Eq. (20), the expression for $\psi$ is obtained as follows

$$
\begin{aligned}
\psi= & c_{4}\left[(r+k)^{m_{1}}-k^{m_{1}}\right]+c_{5}\left[(r+k)^{m_{2}}-k^{m_{2}}\right]+c_{6}\left[(r+k)^{2}-k^{2}\right]+\phi\left\{c_{8}(r+k)^{m_{1}}+\right. \\
& +c_{9}(r+k)^{m_{2}}+c_{4} m_{1}\left(m_{1}-1\right)\left[\frac{(r+k)^{m_{1}+1}}{m_{1}\left(m_{1}+1\right)-\left(1+\lambda_{1}\right) H^{2}}-\frac{k(r+k)^{m_{1}} \ln (r+k)}{2 m_{1}-1}\right] \\
& +c_{5} m_{2}\left(m_{2}-1\right)\left[\frac{(r+k)^{m_{2}+1}}{m_{2}\left(m_{2}+1\right)-\left(1+\lambda_{1}\right) H^{2}}-\frac{k(r+k)^{m_{2}} \ln (r+k)}{2 m_{2}-1}\right] \\
& \left.+2 c_{6}\left[\frac{(r+k)^{3}}{6-\left(1+\lambda_{1}\right) H^{2}}-\frac{k(r+k)^{2}}{2-\left(1+\lambda_{1}\right) H^{2}}\right]\right\} .
\end{aligned}
$$

From Eq. (13), we get the expression for axial velocity as

$$
\begin{aligned}
u= & -m_{1}(r+k)^{m_{1}-1}\left(c_{4}+\phi c_{8}\right)-2 c_{6}(r+k)\left\{1+\phi\left[\frac{3(r+k)}{6-\left(1+\lambda_{1}\right) H^{2}}-\frac{2 k}{2-\left(1+\lambda_{1}\right) H^{2}}\right]\right\} \\
& -m_{2}(r+k)^{m_{2}-1}\left(c_{5}+\phi c_{9}\right)+\left[\frac{k\left(m_{1} \ln (r+k)+1\right)}{2 m_{1}-1}-\frac{\left(m_{1}+1\right)(r+k)}{m_{1}\left(m_{1}+1\right)-\left(1+\lambda_{1}\right) H^{2}}\right] \\
& c_{4} \phi m_{1}\left(m_{1}-1\right)(r+k)^{m_{1}-1}+c_{5} \phi m_{2}\left(m_{2}-1\right)(r+k)^{m_{2}-1}\left[\frac{k\left(m_{2} \ln (r+k)+1\right)}{2 m_{2}-1}\right. \\
& \left.-\frac{\left(m_{2}+1\right)(r+k)}{m_{2}\left(m_{2}+1\right)-\left(1+\lambda_{1}\right) H^{2}}\right] .
\end{aligned}
$$

The pressure rise per wavelength $(\Delta P)$ and skin friction coefficient $\left(C_{f}\right)$ are evaluated using the following expressions:

$$
\begin{aligned}
& \Delta P=\int_{0}^{1} \frac{d p}{d x} d x, \\
& C_{f}=\left(\frac{\partial u}{\partial r}-\frac{u+1}{r+k}\right)_{r=h} .
\end{aligned}
$$

\section{Results and Discussion}

In this section, the solution obtained above is analyzed through graphical representations. Graphs are plotted for the velocity field $(u)$, pressure rise per wavelength $(\Delta P)$, skin friction 
Manjunatha et al.

International Journal of Thermofluid Science and Technology (2020), Volume 7, Issue 2, Paper No. 070203

coefficient $\left(C_{f}\right)$ and streamline patterns to discuss their behavior in the curved channel with variation in the corresponding important parameters. More importantly, we focus on the impact of variation of the curvature parameter $(k)$, variable viscosity $(\phi)$ and magnetic parameter (Hartmann number $H$ ). The values used to obtain the graphs are $\varepsilon=0.2, k=3, \lambda_{1}=1, \quad H=3, \phi=0.2, E_{1}=0.02, E_{2}=0.01, E_{3}=0.01$.
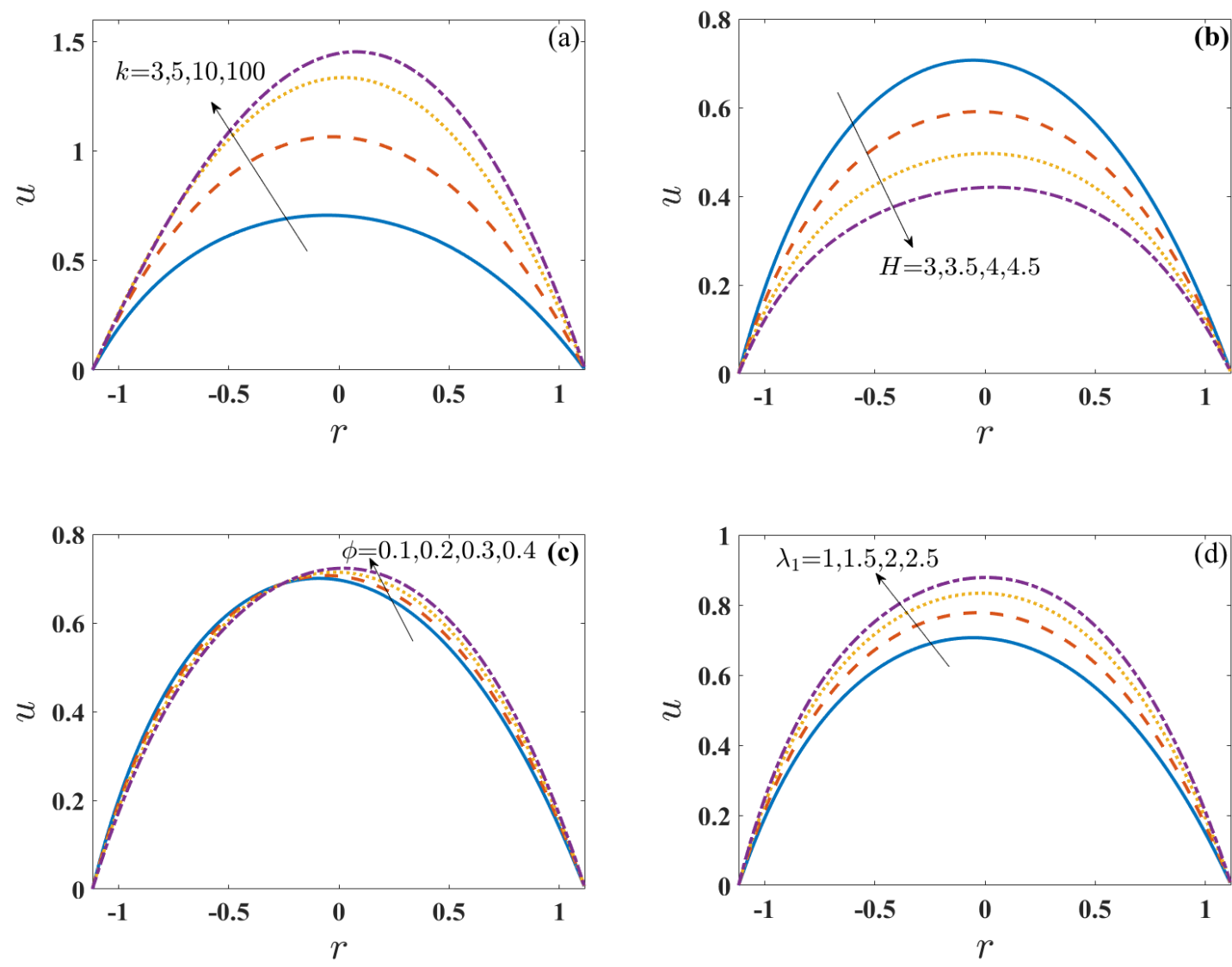

Fig. 2. Variation of axial velocity with (a) $k$, (b) $H$, (c) $\phi$ and (d) $\lambda_{1}$.

Figs. 2(a)-(d) represent the velocity profile in the axial direction. The graphs clearly indicate that the velocity profile displays parabolic geometry, with peak velocity seen in the central section of the channel. Another important observation is that for curved channels, the velocity field is asymmetric about the center of the channel, as opposed to the velocity field for straight channels. However, as seen in Fig. 2(a), the profile shifts towards the channel's center as it becomes straighter, that is, as $k \rightarrow \infty$. The graph also reveals that the fluid velocity rises with the curvature parameter. Fig. 2(b) showcases the influence of Hartmann number on the axial velocity. The velocity sees a decrease with increased magnetic field intensity. In most of the biological fluids, this behavior is due to the development of Lorentz forces owing to exposure to a magnetic field, which opposes the fluid flow. The impact of variable fluid viscosity on the velocity field is sketched in Fig. 2(c). It is seen that the increasing values of variable viscosity reduce the fluid velocity till a point in the channel, after which it increases. This nature can be accounted for by the exponential term defining the variable viscosity. The influence of varying Jeffrey parameter on the velocity of the fluid 
can be observed in Fig. 2(d). The increasing values of $\lambda_{1}$ is found to have an increasing effect on the axial velocity. Moreover, on considering $\lambda_{1}=0$, the corresponding results for a Newtonian fluid can be obtained.
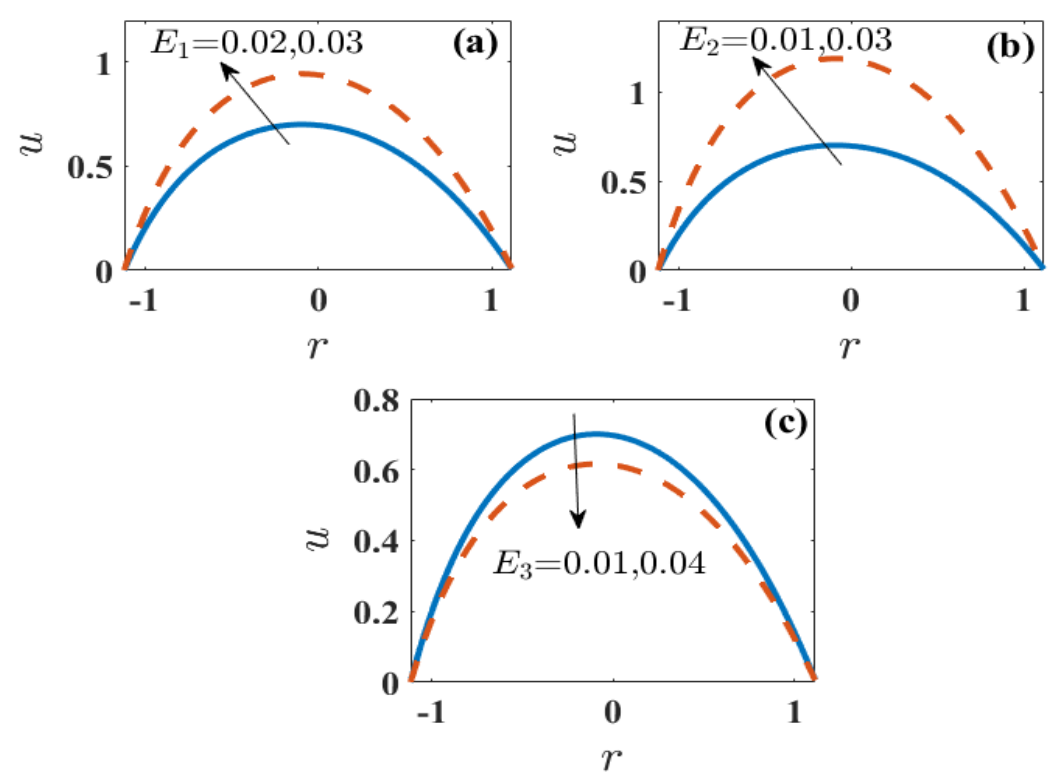

Fig. 3. Variation of axial velocity with (a) $E_{1}$, (b) $E_{2}$ and (c) $E_{3}$.

The effect of wall properties of the curved channel on axial velocity is depicted in Fig. 3 . It is evident from the graph that there is an increase in velocity associated with an increase in $E_{1}$ and $E_{2}$.This can be reasoned by the fact that as the parameters responsible for elasticity and mass per unit area increase, the fluid flows easily, thus boosting its velocity. However, if the damping forces $\left(E_{3}\right)$ are increased, the fluid velocity drops.

The graph for pressure rise versus rate of flow is plotted in Figs. 4(a) and 4(b) for varying values of $H$ and $\phi$. From these figures, a critical value of flow rate can be observed, after which the pressure rise changes from positive to negative. Moreover, the parameters have an opposing effect on the pressure rise before and after this critical value of flow rate. Fig. 4(a) depicts a drop in the pressure rise for increasing values of $H$ above the critical point. An opposite trend is seen in Fig. 4(b) for higher values of $\phi$.
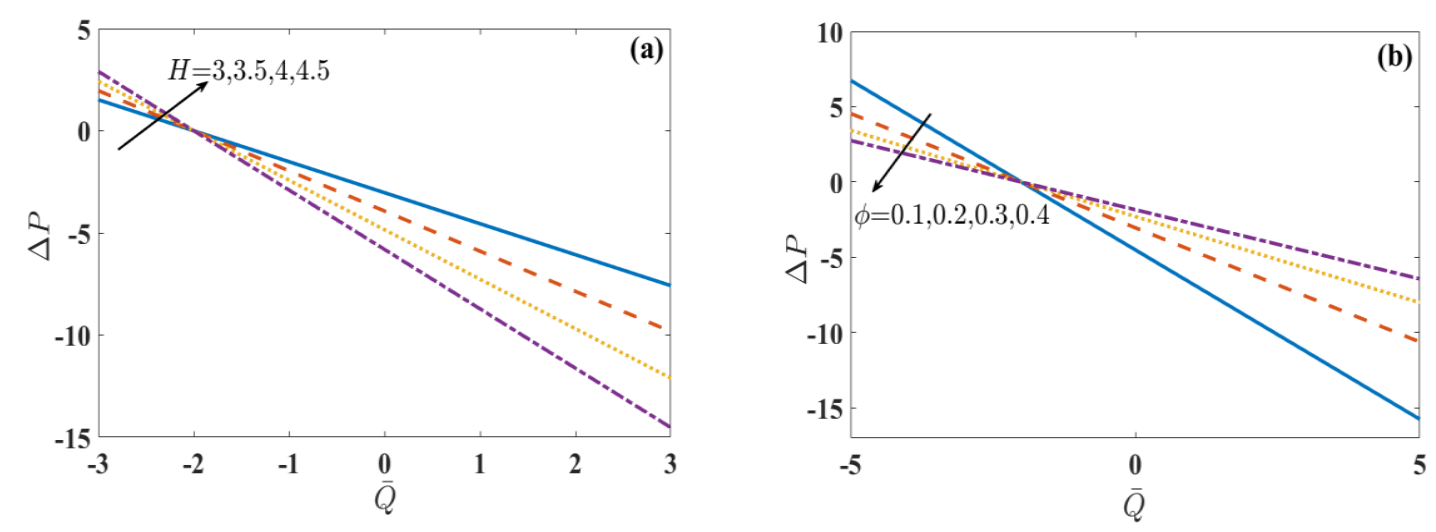
Fig. 4. Variation of pressure rise with (a) $H$ and (b) $\phi$.
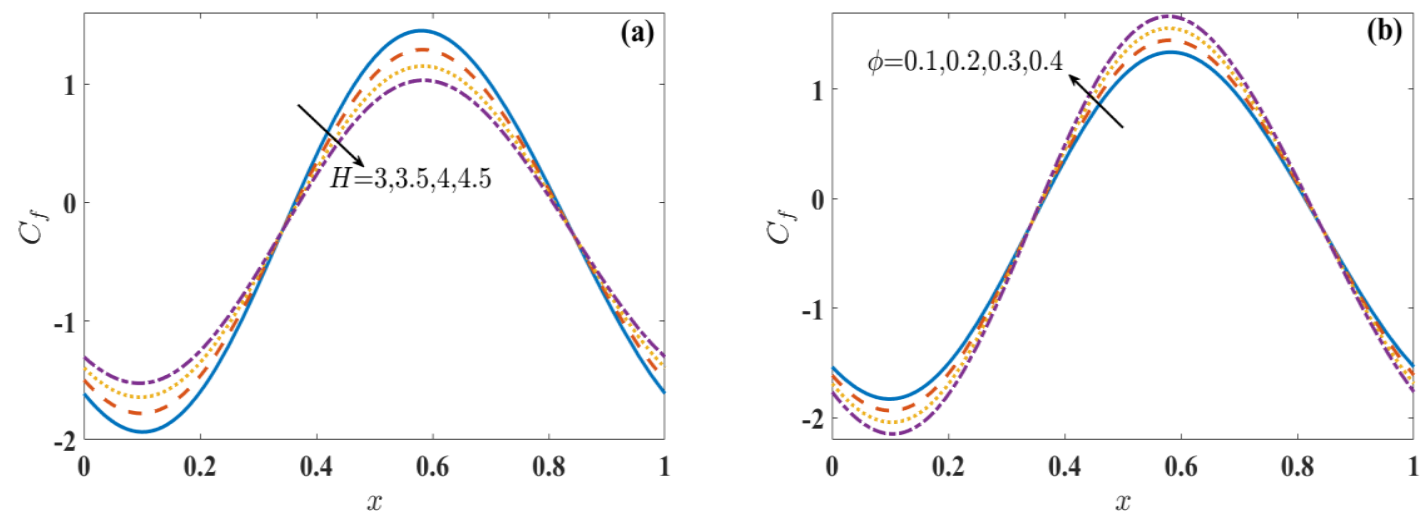

Fig. 5. Variation of skin-friction coefficient with (a) $H$ and (b) $\phi$.

Figs. 5(a) and 5(b) show the impact of $H$ and $\phi$ on the physical quantity of skin friction coefficient. Higher values of $H$ raise the magnitude of skin friction coefficient (see Fig. 5(a)). The effect of increasing values of $\phi$ on $C_{f}$ can be observed in Fig. 5(b), where the magnitude of $C_{f}$ is diminished. It is worth noticing that the effect of these parameters are opposite for $C_{f}>0$ and $C_{f}<0$.

In general, the streamlines assume the form of the boundary walls. However, in some cases, the streamlines were observed to divide and form a circular bolus which gets trapped in the fluid motion. This is the commonly known phenomenon of trapping. The trapped bolus gets encapsulated by the peristaltic wave and moves at the same speed as the wave. Impact of the parameters under consideration on these boluses are depicted in Figs. 6-12. These figures indicate that in a curved channel, the trapped bolus is asymmetric about the center of the channel and divides into asymmetrical parts.

(a)

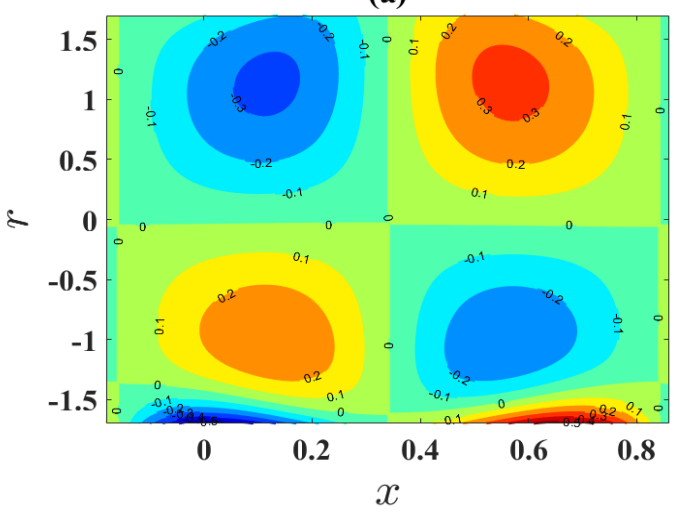

(b)

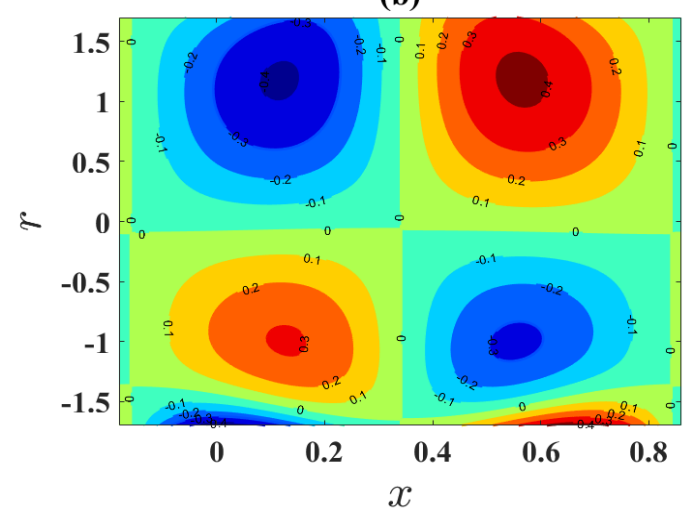

Fig. 6. Streamlines for (a) $k=3$ and (b) $k=3.5$. 
Manjunatha et al.

International Journal of Thermofluid Science and Technology (2020), Volume 7, Issue 2, Paper No. 070203
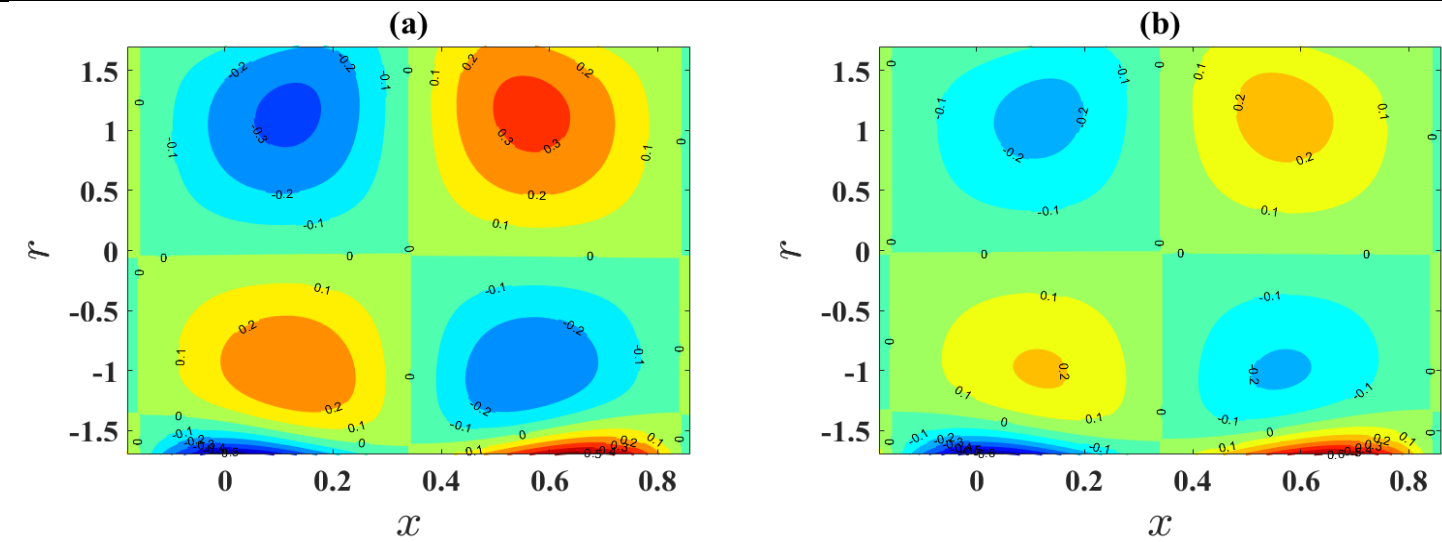

Fig. 7. Streamlines for (a) $H=3$ and (b) $H=4$.

(a)

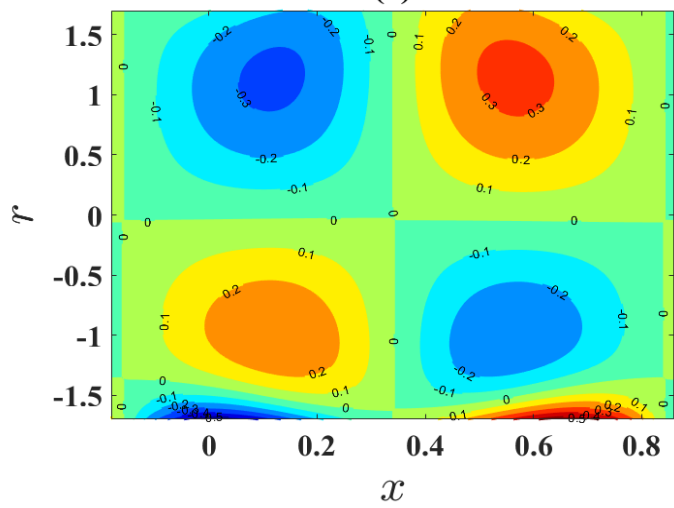

(b)

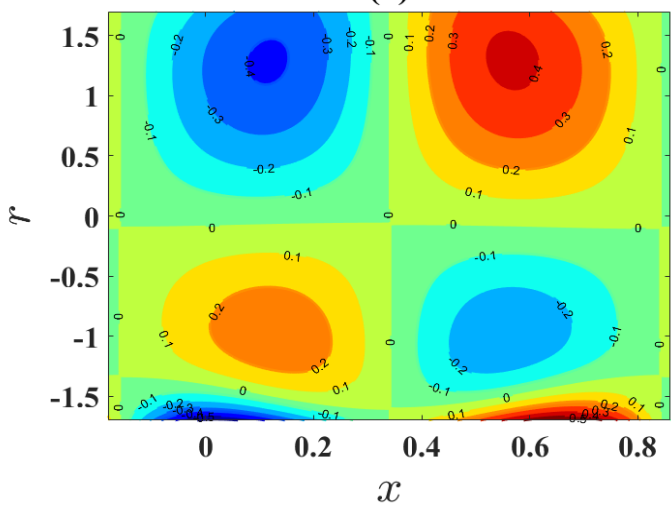

Fig. 8. Streamlines for (a) $\phi=0.01$ and (b) $\phi=0.02$.
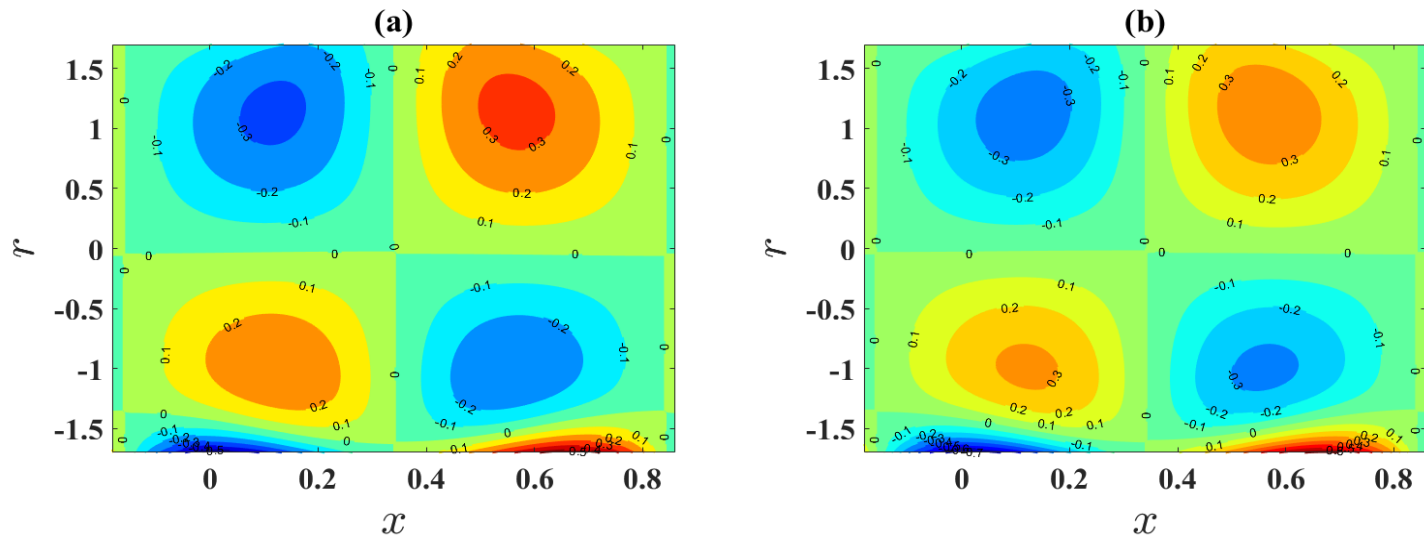

Fig. 9. Streamlines for (a) $\lambda_{1}=1$ and (b) $\lambda_{1}=1.5$. 
(a)

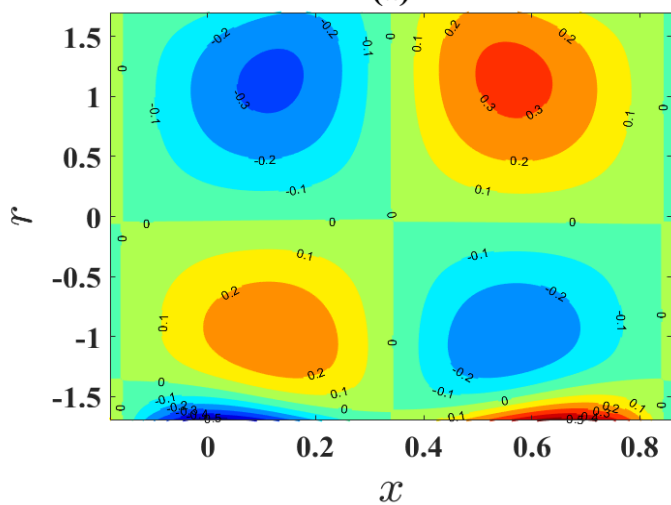

(b)

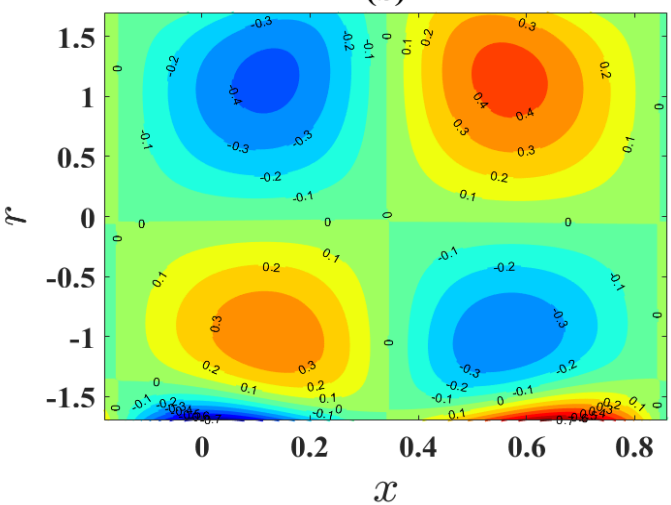

Fig. 10. Streamlines for (a) $E_{1}=0.02$ and (b) $E_{1}=0.03$.

(a)

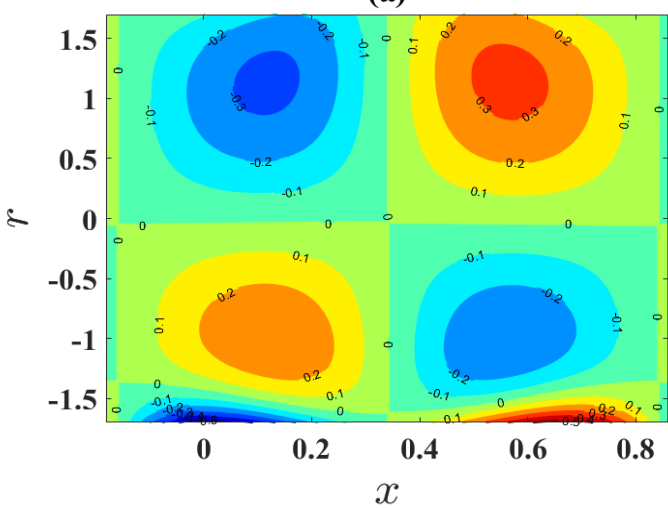

(b)

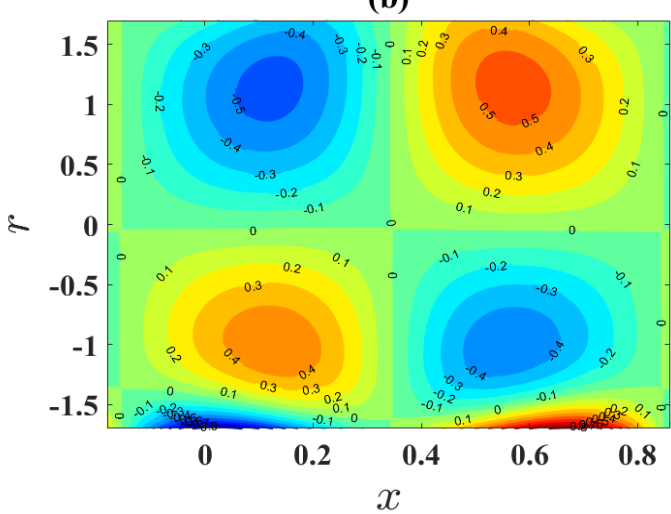

Fig. 11. Streamlines for (a) $E_{2}=0.01$ and (b) $E_{2}=0.03$.

(a)

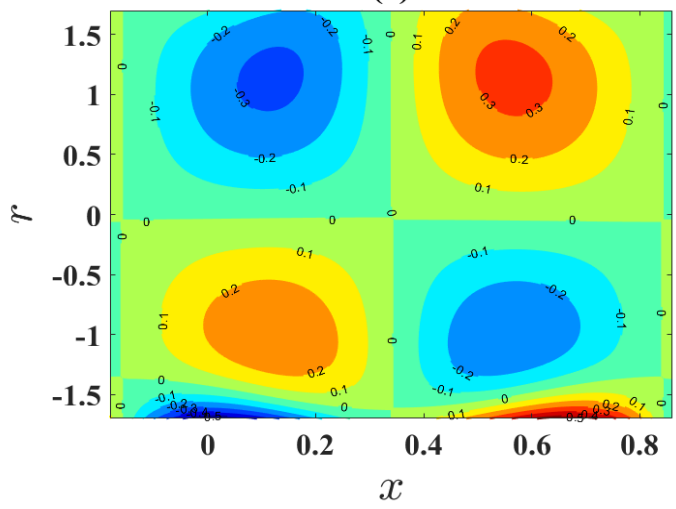

(b)

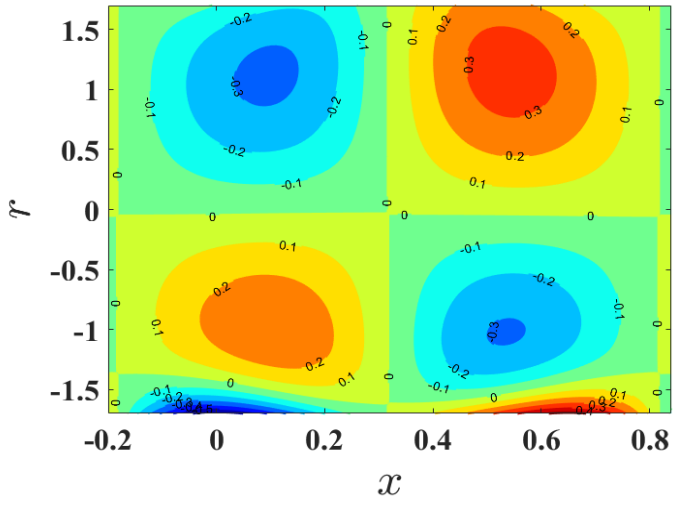

Fig. 12. Streamlines for (a) $E_{3}=0.01$ and (b) $E_{3}=0.04$.

From Fig. 6, the trapped bolus expands with an enhancement in the curvature of the channel. Physically, this signifies that the phenomenon of trapping has a higher likelihood of occurrence in a straight channel compared to a curved channel.

Fig. 7 describes the impact on the streamlines pattern due to variation in $H$. It shows a shrinking of the trapped bolus when the Hartmann number is increased. Fig. 8 elucidates the influence of $\phi$ on trapping phenomena. It is observed that the trapped bolus grows in the upper half of the curved channel. Meanwhile, in the lower half of the channel, the streamlines 
Manjunatha et al.

International Journal of Thermofluid Science and Technology (2020), Volume 7, Issue 2, Paper No. 070203

get compressed and the bolus size decreases for higher values of $\phi$. The streamlines plotted in Fig. 9 for varying values of the Jeffrey parameter indicate that it has an expansion effect on the volume of the trapped bolus.

Figs. 10-12 shows the variation in the bolus volume due to the wall properties $E_{1}, E_{2}$ and $E_{3}$. The observations reveal that $E_{1}$ and $E_{2}$ increase the volume of the trapped bolus whereas $E_{3}$ decreases the volume.

\section{Conclusion}

The current investigations attempt to explain the impact of variations in viscosity on the peristaltic mechanism exhibited by a non-Newtonian Jeffrey fluid through a channel with significant curvature when exposed to an external magnetic field with radial orientation. Furthermore, the channel walls considered were compliant. The fluid viscosity was assumed to be varying in proportion to the width of the channel. Perturbed solution for velocity along the axis has been obtained for small values of variable viscosity $\phi$. Some of the important outcomes of the investigation are outlined below:

- Velocity is asymmetric about the center of a curved channel. However, the velocity profile shifts to the center as the channel becomes straighter.

- Variable viscosity has a decreasing impact on fluid velocity closer to the lower channel wall.

- The pressure rise enhances above the critical value of flow rate with higher values of Hartmann number.

- The bolus confined in the fluid motion expands in proportion to higher curvature parameter and Jeffrey parameter values.

- Variable viscosity has a dual effect on the bolus size, where it is enhanced in the upper half but diminishes in the lower half of the curved channel.

- The axial velocity and bolus size is increased with the wall property parameters $E_{1}$ and $E_{2}$, whereas $E_{3}$ decreases them.

\section{Acknowledgement}

The authors would like to thank the anonymous reviewers for their constructive and helpful comments that have led to a substantial improvement in the paper.

\section{References}

[1] Raju, K. K., Devanathan, R., Peristaltic motion of a non-Newtonian fluid. Rheologica Acta. 11 (1972) 170-178.

[2] Rajashekhar, C., Manjunatha, G., Prasad, K. V., Divya, B. B., Vaidya, H., Peristaltic transport of two-layered blood flow using Herschel-Bulkley Model. Cogent Engineering. 5 (2018) 1495592.

[3] Vaidya, H., Rajashekhar, C., Manjunatha, G., Prasad, K. V., Effects of Heat Transfer on Peristaltic Transport of a Bingham Fluid through an Inclined Tube with Different Wave Forms. In Defect and Diffusion Forum. 392 (2019) 158-177. 
Manjunatha et al.

International Journal of Thermofluid Science and Technology (2020), Volume 7, Issue 2, Paper No. 070203

[4] Vaidya, H., Gudekote, M., Choudhari, R., K.V. Prasad, Role of slip and heat transfer on peristaltic transport of Herschel-Bulkley fluid through an elastic tube. Multidiscipline Modeling in Materials and Structures. 14 (2018) 940-959.

[5] Vaidya, H., Rajashekhar, C., Manjunatha, G., Prasad, K. V., Rheological properties and peristalsis of Rabinowitsch fluid through compliant porous walls in an inclined channel. Journal of Nanofluids. 8 (2018) 970-979.

[6] Balocco C., Petrone G., Heat and moisture transfer investigation of surface building materials. Mathematical Modelling of Engineering Problems. 5 (2018) 146-152.

[7] Camaraza-Medina, Y., Rubio-Gonzales, Á. M., Cruz-Fonticiella, O. M., García-Morales, O. F., Simplified analysis of heat transfer through a finned tube bundle in air cooled condenser. Mathematical Modelling of Engineering Problems. 5 (2018) 237-242.

[8] Ali, N., Sajid, M., Hayat, T., Long wavelength flow analysis in a curved channel. Zeitschrift für Naturforschung A. 65 (2010) 191-196.

[9] Ali, N., Sajid, M., Abbas, Z., Javed, T., Non-Newtonian fluid flow induced by peristaltic waves in a curved channel. European Journal of Mechanics-B/Fluids. 29 (2010) 387-394.

[10] Ali, N., Sajid, M., Javed, T., Abbas, Z., Heat transfer analysis of peristaltic flow in a curved channel. International Journal of Heat and Mass Transfer. 53 (2010) 3319-3325.

[11] Hayat, T., Javed, M., \& Hendi, A. A., Peristaltic transport of viscous fluid in a curved channel with compliant walls. International Journal of Heat and Mass Transfer. 54 (2011) 1615-1621.

[12] Hayat, T., Abbasi, F. M., Ahmad, B., Alsaedi, A., Peristaltic transport of Carreau-Yasuda fluid in a curved channel with slip effects. PloS one. 9 (2014) e95070.

[13] Narla, V. K., Prasad, K. M., \& Ramanamurthy, J. V., Peristaltic transport of Jeffrey nanofluid in curved channels. Procedia Engineering. 127 (2015) 869-876.

[14] Hina, S., Mustafa, M., Hayat, T., Alotaibi, N. D., On peristaltic motion of pseudoplastic fluid in a curved channel with heat/mass transfer and wall properties. Applied Mathematics and Computation. 263 (2015) 378-391.

[15] Hayat, T., Rafiq, M., Alsaadi, F., Ayub, M., Soret and Dufour effects on peristaltic transport in curved channel with radial magnetic field and convective conditions. Journal of Magnetism and Magnetic Materials. 405 (2016) 358-369.

[16] Shehzad, S. A., Abbasi, F. M., Hayat, T., Alsaadi, F., Mousa, G., Peristalsis in a curved channel with slip condition and radial magnetic field. International Journal of Heat and Mass Transfer. 91 (2015) 562-569.

[17] Hayat, T., Noreen, S., Alsaedi, A., Effect of an induced magnetic field on peristaltic flow of non-Newtonian fluid in a curved channel. Journal of Mechanics in Medicine and Biology. 12 (2012) 1250058.

[18] Abbasi, F. M., Hayat, T., Alsaedi, A., Numerical analysis for MHD peristaltic transport of Carreau-Yasuda fluid in a curved channel with Hall effects. Journal of Magnetism and Magnetic Materials. 382 (2015) 104-110.

[19] Hayat, T., Farooq, S., Ahmad, B., Alsaedi, A., Homogeneous-heterogeneous reactions and heat source/sink effects in MHD peristaltic flow of micropolar fluid with Newtonian heating in a curved channel. Journal of Molecular Liquids. 223 (2016) 469-488.

[20] Hayat, T., Tanveer, A., Alsaedi, A., Numerical analysis of partial slip on peristalsis of MHD Jeffery nanofluid in curved channel with porous space. Journal of Molecular Liquids. 224 (2016) 944-953.

[21] Hayat, T., Tanveer, A., Alsaadi, F., Mousa, G., Impact of radial magnetic field on peristalsis in curved channel with convective boundary conditions. Journal of Magnetism and Magnetic Materials. 403 (2016) 47-59.

[22] Vasanthakumari R., Pondy P., Mixed convection of silver and titanium dioxide nanofluids along inclined stretching sheet in presence of MHD with heat generation and suction effect. Mathematical Modelling of Engineering Problems. 5 (2018) 123-129. 
Manjunatha et al.

International Journal of Thermofluid Science and Technology (2020), Volume 7, Issue 2, Paper No. 070203

[23] Nayakar, R., Mahabaleshwar, U.S., Vinaykumar, P.N., Lorenzini, G., Baleanu, D., Nonlinear stretching/shrinking cooling of a sheet involving an MHD Walters' liquid B with suction. Mathematical Modelling of Engineering Problems. 6 (2019) 343-348.

[24] Singh, J.K., Seth, G.S., Rohidas, P., Impacts of time varying wall temperature and concentration on MHD free convective flow of a rotating fluid due to moving free-stream with hall and ion-slip currents. International Journal of Thermofluid Science and Technology. 6 (2019) 19060301.

[25] Lachiheb, M., On the effect of variable viscosity on the peristaltic transport of a Newtonian fluid in an asymmetric channel. Canadian Journal of Physics. 94 (2016) 320-327.

[26] Awais, M., Bukhari, U., Ali, A., Yasmin, H., Convective and peristaltic viscous fluid flow with variable viscosity. Journal of Engineering Thermophysics. 26 (2017) 69-78.

[27] Rajashekhar, C., Manjunatha, G., Vaidya, H., Divya, B., Prasad, K., Peristaltic flow of Casson liquid in an inclined porous tube with convective boundary conditions and variable liquid properties. Frontiers in Heat and Mass Transfer (FHMT). 11 (2018).

[28] Vaidya, H., Choudhari, R., Gudekote, M., Prasad, K. V., Makinde, O. D., Sreenadh, S., Peristaltic Motion of Non-Newtonian Fluid with Variable Liquid Properties in a Convectively Heated Non-Uniform Tube: Rabinowitsch Fluid Model. Journal of Enhanced Heat Transfer. (2019)

[29] Divya, B., Manjunatha, G., Rajashekhar, C., Hanumesh, V., Prasad, K., Impact of variable liquid properties on Peristaltic mechanism of convectively heated Jeffrey fluid in a slippery elastic tube. Frontiers in Heat and Mass Transfer (FHMT). 12 (2018).

[30] Manjunatha, G., Rajashekhar, C., Vaidya, H., Prasad, K. V., Peristaltic mechanism of Bingham liquid in a convectively heated porous tube in the presence of variable liquid properties. Special Topics \& Reviews in Porous Media: An International Journal. 10 (2019).

[31] Vaidya, H., Rajashekhar, C., Manjunatha, G., Prasad, K. V., Peristaltic mechanism of a Rabinowitsch fluid in an inclined channel with complaint wall and variable liquid properties. Journal of the Brazilian Society of Mechanical Sciences and Engineering. 41 (2019) 52.

[32] Manjunatha, G., Rajashekhar, C., Prasad, K.V., Vaidya, H., Peristaltic flow of a Jeffery fluid over a porous conduit in the presence of variable liquid properties and convective boundary. International Journal of Thermofluid Science and Technology. 6 (2019) 19060201.

[33] Manjunatha, G., Rajashekhar, C., Vaidya, H., Prasad, K.V., Saraswati, Divya B.B., Heat Transfer Analysis on Peristaltic Transport of a Jeffery Fluid in an Inclined Elastic Tube with Porous Walls. International Journal of Thermofluid Science and Technology. 7 (2020) 20070101.

[34] Hayat, T., Hina, S., Hendi, A. A., Asghar, S., Effect of wall properties on the peristaltic flow of a third-grade fluid in a curved channel with heat and mass transfer. International Journal of Heat and Mass Transfer. 54 (2011) 5126-5136.

[35] Ali, N., Hussain, Q., Hayat, T., Asghar, S. (2008). Slip effects on the peristaltic transport of MHD fluid with variable viscosity. Physics Letters A. 372(2008) 1477-1489.

\section{Appendix}


Manjunatha et al.

International Journal of Thermofluid Science and Technology (2020), Volume 7, Issue 2, Paper No. 070203

$$
\begin{aligned}
& m_{1}=\frac{1+\sqrt{1+4\left(1+\lambda_{1}\right) H^{2}}}{2}, \quad m_{2}=\frac{1-\sqrt{1+4\left(1+\lambda_{1}\right) H^{2}}}{2}, \\
& c_{1}=-8 \pi^{3} \varepsilon k\left[\left(E_{1}+E_{2}\right) \cos (2 \pi(x-t))-\frac{E_{3}}{2 \pi} \sin (2 \pi(x-t))\right], c_{3}=c_{1}\left(1+\lambda_{1}\right), \\
& c_{4}=\frac{-2 c_{6}}{m_{2}}\left[\frac{(-h+k)^{2-m_{2}}(h+k)^{m_{2}-m_{1}}\left[(-h+k)^{m_{1}-2}(h+k)^{2-m_{1}}-1\right]}{1-(h+k)^{m_{2}-m_{1}}(-h+k)^{m_{1}-m_{2}}}+(h+k)^{2-m_{1}}\right] \text {, } \\
& c_{5}=\frac{2 c_{6}}{m_{2}}(-h+k)^{2-m_{2}}\left[\frac{(h+k)^{2-m_{1}}(-h+k)^{m_{1}-2}-1}{1-(h+k)^{m_{2}-m_{1}}(-h+k)^{m_{1}-m_{2}}}\right], c_{6}=\frac{c_{1}\left(1+\lambda_{1}\right)}{2\left(-2+\left(1+\lambda_{1}\right) H^{2}\right)}, c_{7}=\frac{\frac{c_{3} k^{2}}{2}+c_{2}}{\left(1+\lambda_{1}\right) H^{2}}, \\
& c_{8}=\frac{\left(\frac{-h+k}{h+k}\right)^{m_{2}-1}\left[c_{10} m_{1}\left(m_{1}-1\right)+c_{11} m_{2}\left(m_{2}-1\right)+c_{12}\right]}{m_{1}(-h+k)^{m_{1}-1}-m_{1}(h+k)^{m_{1}-m_{2}}(-h+k)^{m_{2}-1}}-\frac{c_{13} m_{1}\left(m_{1}-1\right)+c_{14} m_{2}\left(m_{2}-1\right)+c_{15}}{m_{1}(-h+k)^{m_{1}-1}-m_{1}(h+k)^{m_{1}-m_{2}}(-h+k)^{m_{2}-1}}, \\
& c_{9}=-\left[\frac{c_{10} m_{1}\left(m_{1}-1\right)+c_{11} m_{2}\left(m_{2}-1\right)+c_{8} m_{1}(h+k)^{m_{1}-1}+c_{12}}{m_{2}(h+k)^{m_{2}-1}}\right], \\
& c_{10}=c_{4}\left[\frac{\left(m_{1}+1\right)(h+k)^{m_{1}}}{m_{1}\left(m_{1}+1\right)-\left(1+\lambda_{1}\right) H^{2}}-\frac{k(h+k)^{m_{1}-1}\left(m_{1} \ln (h+k)+1\right)}{2 m_{1}-1}\right], \\
& c_{11}=c_{5}\left[\frac{\left(m_{2}+1\right)(h+k)^{m_{2}}}{m_{2}\left(m_{2}+1\right)-\left(1+\lambda_{1}\right) H^{2}}-\frac{k(h+k)^{m_{2}-1}\left(m_{2} \ln (h+k)+1\right)}{2 m_{2}-1}\right], \\
& c_{12}=2 c_{6}\left[\frac{3(h+k)^{2}}{6-\left(1+\lambda_{1}\right) H^{2}}-\frac{2 k(h+k)}{2-\left(1+\lambda_{1}\right) H^{2}}\right] \text {, } \\
& c_{13}=c_{4}\left[\frac{\left(m_{1}+1\right)(-h+k)^{m_{1}}}{m_{1}\left(m_{1}+1\right)-\left(1+\lambda_{1}\right) H^{2}}-\frac{k(-h+k)^{m_{1}-1} m_{1} \ln (-h+k)}{2 m_{1}-1}-\frac{k(-h+k)^{m_{1}-1}}{2 m_{1}-1}\right] \text {, } \\
& c_{14}=c_{5}\left[\frac{\left(m_{2}+1\right)(-h+k)^{m_{2}}}{m_{2}\left(m_{2}+1\right)-\left(1+\lambda_{1}\right) H^{2}}-\frac{k(-h+k)^{m_{2}-1} m_{2} \ln (-h+k)}{2 m_{2}-1}-\frac{k(-h+k)^{m_{2}-1}}{2 m_{2}-1}\right], \\
& c_{15}=2 c_{6}\left[\frac{3(-h+k)^{2}}{6-\left(1+\lambda_{1}\right) H^{2}}-\frac{2 k(-h+k)}{2-\left(1+\lambda_{1}\right) H^{2}}\right] \text {. }
\end{aligned}
$$

